\title{
DOA Estimation of LFM Signal Based on Single-Source Time-Frequency Points Selection Algorithm by Using the Hough Transform
}

\author{
Weike ZHANG, Kaibo CUI, Weiwei WU, Tao XIE, Naichang YUAN \\ State Key Laboratory of Complex Electromagnetic Environment Effects on Electronics and Information System, \\ National University of Defense Technology, Changsha, 410073, China \\ xdwdz2010@163.com
}

Submitted March 25, 2018 / Accepted December 22, 2018

\begin{abstract}
Direction of arrival (DOA) estimation performance may degrade substantially when linear frequency modulation (LFM) signals are spectrally-overlapped in time-frequency (TF) domain. In order to solve this problem, the single-source TF points selection algorithm based on Wigner-Ville distribution (WVD) and Hough transform is studied in this paper. Firstly, the signal intersections in TF domain can be solved based on the Hough transform. Secondly, by removing multiple-source TF points at intersections according to the empirical threshold value which is calculated by using the statistical experiment method, we can get single-source TF points set. Then, based on the Euclidean distance operator, single-source TF points set belonging to each signal can be obtained according to the property that TF points of the same signal have the same eigenvector. Finally, the averaged spatial TF distribution matrix is constructed and DOA estimation is realized based on the multiple signal classification (MUSIC) algorithm. In this way, the proposed algorithm can resolve the TF non-disjoint LFM signals because it can automatically select single-source TF points set of each signal. Simulation results illustrate that the proposed algorithm possesses higher angular resolution and has pretty good DOA estimation precision compared with existing algorithms.
\end{abstract}

\section{Keywords}

LFM signal, DOA estimation, time-frequency analysis, single-source time-frequency points, Hough transform, WVD

\section{Introduction}

Direction of arrival (DOA) estimation is widely used at radar, speech, sonar, communication and other detection equipment [1-5]. For the narrowband signals, traditional subspace algorithms which include the multiple signal classification (MUSIC) algorithm [1] and the estimation of signal parameters via rotational invariance techniques
(ESPRIT) [2] have been verified as efficient estimation techniques. Linear frequency modulation (LFM) signals have many good characteristics, such as carrying a large amount of information and possessing better detection performance, which makes it being widely used in electronic countermeasures. However, subspace-based algorithms have limitations with regard to dealing with LFM signals which are wideband and non-stationary. So it is very important to study the DOA estimation of LFM signals. TF analysis tools are widely used to deal with LFM signals because it can take full advantage of the non-stationary property of LFM signals [6]. Previous TF analysis is mainly applied to signal waveform restoration in blind source separation. Belouchrani et al. firstly proposed the concept of spatial time-frequency distribution matrix (STFD) based on Wigner-Ville distribution (WVD) [7] and then a series of DOA estimation algorithms based on STFD matrix have been proposed [8-11]. These algorithms use the STFD matrix to replace the covariance matrix in the MUSIC method, which forms the TF-MUSIC algorithm. Compared with the MUSIC algorithm, TF-MUSIC can provide better DOA estimation performance since the TF distribution spreads the noise power while localizing the source energy in TF domain. Early TF-MUSIC algorithms are based on the narrowband signal model, and gradually extend to the wideband signal model [12], [13].

In order to construct the STFD matrix, it is necessary to select the single-source TF points of each signal in TF domain. When the signals are TF-disjoint, many references have proposed efficient single-source TF points selection methods [14], [15]. The reference [14] obtained the singlesource TF points by using the clustering algorithm. In [15], Heidenreich applied morphological image processing to detect instantaneous frequency segments of each signal. The detected IF segments are combined based on a bootstrap resampling technique, and the linking IF segments belonging to a single source are then used for DOA estimation. Although these two methods all can correct select the single-source TF points, they only can be used when the signals are TF-disjoint. 
When the signals are non-disjoint (i.e., spectrallyoverlapped) in TF domain, the studies of single-source TF point selection algorithms are not very full. In [16-18], the authors extracted single-source TF points based on STFT distribution. However, due to the contradiction between time resolution and frequency resolution, the description accuracy of STFT is low in TF domain, which results in poor DOA estimation performance. In addition, the reference [19] used the forward-backward spatial smoothing (FBSS) algorithm to eliminate the influence of multiplesource TF points in TF domain. Although the algorithm can estimate the DOAs of LFM signals, the estimation accuracy is low owing to the spatial smoothing process. Due to the fact that WVD can more accurately describe the TF distribution of LFM signals, the WVD has been widely used to select single-source TF points. [20-21] explained how to avoid the cross-term TF points and obtain the autoterm TF points, but single-source TF points selection problems are not involved. [22] proposed single-source TF points selection algorithm based on improved clustering algorithm. Although the algorithm achieves blind source separation of signals, it is just used in the speech signal process. In this paper, we mainly study the DOA estimation of LFM signals.

When multiple LFM signals are non-disjoint in TF domain, the TF domain contains noise TF points, crossterm TF points and auto-term TF points. The noise TF points and cross-term TF points can be eliminated based on the corresponding algorithms [23]. The auto-term TF points contain single-source TF points and multiple-source TF points. According to [19], by carrying singular value decomposition (SVD) of the STFD matrix, we can obtain the signal subspace matrix. The matrix is column full-rank at the single-source TF points and the rank is equal to the number of signals. At the multiple-source TF points, the signal subspace matrix behaves as the rank deficiency, which means that the rank is less than the number of signals. Theoretically, if the number of signals is known, then single-source TF points can be selected according to the size of the signal subspace matrix rank by traversing the entire TF domain. However, in practice, the number of signals is unknown. Although the number of signals can be estimated based on the information theory criterion, the estimation accuracy is affected by many factors. Once the estimation is wrong, the subsequent DOA estimation will fail, and this method is very complicated. Since the distribution shape of LFM signals is multiple straight lines in TF domain, which reminds us of the Hough transform that detects straight lines in the image processing. Based on the Hough transform, the intersections of signals in TF domain can be obtained. Then, taking the intersection as the center, multiple-source TF points can be removed based on the empirical threshold value which can be calculated by using the statistical experiment method, and single-source TF points are reserved. Then, according to the property that the TF point of the same signal have the same eigenvector, single-source TF points belonging to each signal can be obtained. Finally, the STFD matrix is constructed for each signal, and DOA estimation is performed based on the MUSIC algorithm.

The remainder of the paper is organized as follows: Section 2 formulates the WVD model of LFM signals. Section 3 illustrates how to select single-source TF points. The numerical simulations and discussions are carried in Sec. 4. Section 5 performs experimental verification and Section 6 concludes the paper.

\section{WVD Model of LFM Signals}

In this paper, we consider a uniform linear array (ULA) has $M$ sensors. Assuming that $N$ far-field LFM signals imping on the ULA, and the array element spacing is $d$. The LFM signal $s_{n}(t)$ is defined as

$$
s_{n}(t)=\exp \left(\mathrm{j} 2 \pi f_{n 0} t+\mathrm{j} \pi \gamma_{n} t^{2}\right)
$$

where $f_{n 0}$ and $\gamma_{n}$ denote the initial frequency and modulated frequency of the $n$th signal respectively. $f_{n}(t)=f_{n 0}+\gamma_{n} t$ is the instantaneous frequency. If $\gamma_{n}=0$, the LFM signal becomes common single-frequency signal.

Assuming that the first element is set as the phase reference point which is located at the coordinate origin, then, the time delay of the $n$th signal on the $m$ th sensor respecting to the reference sensor can be expressed as

$$
\tau_{n m}=-\frac{(m-1) d \cos \left(\theta_{n}\right)}{c} .
$$

So the received signals of the $m$ th sensor is written as

$$
\begin{aligned}
S_{m}(t) & =\sum_{n=1}^{N} s_{n}\left(t-\tau_{n m}\right) \\
& =\sum_{n=1}^{N} \exp \left[\mathrm{j} 2 \pi f_{n 0}\left(t-\tau_{n m}\right)+\mathrm{j} \pi \gamma_{n}\left(t-\tau_{n m}\right)^{2}\right] .
\end{aligned}
$$

Considering the effects of additive white Gaussian noise $n_{m}(t)$. The $m$ th sensor output is

$$
x_{m}(t)=S_{m}(t)+n_{m}(t) .
$$

More compactly, (4) can be modeled as

$$
\mathbf{X}(t)=\mathbf{A S}(t)+\mathbf{N}(t) \text {. }
$$

$\mathbf{X}(t)$ is $M \times 1$ array output vector and $\mathbf{S}(t)$ is $N \times 1$ LFM signals vector. $\mathbf{N}(t)$ is $M \times 1$ noise vector. $\mathbf{A}=\left[\mathbf{a}_{1}\left(f_{1}(t)\right), \cdots, \mathbf{a}_{N}\left(f_{N}(t)\right)\right]$ is the manifold vector and $\mathbf{a}_{n}\left(f_{n}(t)\right)=\left[\exp \left(-\mathrm{j} f_{n}(t)\right) \tau_{n 1}, \cdots, \exp \left(-\mathrm{j} f_{n}(t)\right) \tau_{n M}\right]^{\mathrm{T}}$ is the steering vector of the $n$th source at time $t$, where $(\cdot)^{\mathrm{T}}$ is the transpose operator.

In 1932, Wigner proposed the WVD in quantum mechanics field [24] and Ville introduced WVD to signal analysis field in 1948. WVD is a very effective TF analysis tool. For the signal $s(t), r_{s}(t, \tau)=s(t+\tau) s^{*}(t-\tau)$ is the instantaneous autocorrelation of $s(t)$, where $\tau$ denotes the time delay and $(\cdot)^{*}$ is the complex conjugate operator. The 
continuous WVD is defined as the Fourier transform of $r_{s}(t, \tau)$,

$$
\operatorname{WVD}_{s}(t, f)=\int_{-\infty}^{+\infty} s(t+\tau) s^{*}(t-\tau) \mathrm{e}^{-\mathrm{j} 4 \pi f \tau} \mathrm{d} \tau .
$$

In the actual system, by sampling $X(t)$ at the sampling rate $f_{\mathrm{s}}$, we can obtain the discrete vector $\mathbf{X}(k)$ from $X(t)$. Assuming $L$ is the sampling data length, then discrete pseudo WVD (DPWVD) of $\mathbf{X}(k)$ can be expressed as

$$
\mathbf{D}_{\mathrm{XX}}(k, f)=2 \sum_{l=-(L-1) / 2}^{(L-1) / 2} \mathbf{X}(k+l) \mathbf{X}^{*}(k-l) \exp (-\mathrm{j} 4 \pi f l) \text {. }
$$

In the free of noise, substituting (5) into (7), we can obtain

$$
\mathbf{D}_{\mathrm{XX}}(k, f)=\mathbf{A}(k, f) \mathbf{D}_{\mathrm{SS}}(k, f) \mathbf{A}^{\mathrm{H}}(k, f)
$$

where $\mathbf{D}_{\mathrm{XX}}$ and $\mathbf{D}_{\mathrm{SS}}$ are array output STFD matrix and signal STFD matrix, respectively.

\section{Single-source TF Points Selection Method Based on Hough Transform}

The non-disjoint TF distribution of LFM signals in TF domain can be described as Fig. 1. Figure 1(b) represents that signals are largely overlapped in TF domain, and the reference [19] has proposed efficient DOA estimation method. Therefore, we mainly study that signals are tiny non-disjoint in TF domain, which means that there always exist most TF points that are only associated with this signal, as is shown in Fig. 1(a).

We consider that two LFM signals $S_{1}(t), S_{2}(t)$ impinge on the ULA, then the signal STFD matrix $\mathbf{D}_{\mathrm{SS}}$ can be expressed as

$$
\mathbf{D}_{S S}(t, f)=\left(\begin{array}{ll}
\mathbf{D}_{S_{1} S_{1}}(t, f) & \mathbf{D}_{S_{1} S_{2}}(t, f) \\
\mathbf{D}_{S_{2} S_{1}}(t, f) & \mathbf{D}_{S_{2} S_{2}}(t, f)
\end{array}\right)
$$

where $\mathbf{D}_{\mathrm{S}_{1} \mathrm{~S}_{1}}(t, f)$ and $\mathbf{D}_{\mathrm{S}_{2} \mathrm{~S}_{2}}(t, f)$ denote the signal auto-term TF points, $\mathbf{D}_{\mathrm{S}_{1} \mathrm{~S}_{2}}(t, f)$ and $\mathbf{D}_{\mathrm{S}_{2} \mathrm{~S}_{1}}(t, f)$ denote the signal crossterm TF points. According to (8) and (9), $\mathbf{D}_{\mathrm{XX}}$ can be written as

$$
\mathbf{D}_{\mathrm{XX}}(t, f)=\mathbf{A}(t, f)\left(\begin{array}{ll}
\mathbf{D}_{\mathrm{S}_{1} \mathrm{~S}_{1}} & \mathbf{D}_{\mathrm{S}_{1} \mathrm{~S}_{2}} \\
\mathbf{D}_{\mathrm{S}_{2} \mathrm{~S}_{1}} & \mathbf{D}_{\mathrm{S}_{2} \mathrm{~S}_{2}}
\end{array}\right) \mathbf{A}^{\mathrm{H}}(t, f) .
$$

Equation (10) represents the distribution of array output STFD matrix, which includes cross-term and autoterm. Auto-term consists of single-source TF points and multiple-source TF points. The cross-term is caused by the bilinear operation of the WVD transform.

Figure 2 shows different types of TF points distribution in the smoothed TF domain. Type1 represents noiseterm TF points and Type 2 represents cross-term TF points. Type 3 represents multiple-source TF points and Type4 represents single-source TF points. Among four types of

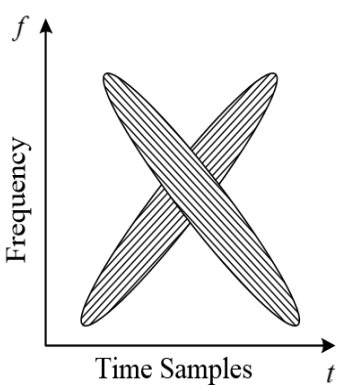

(a)

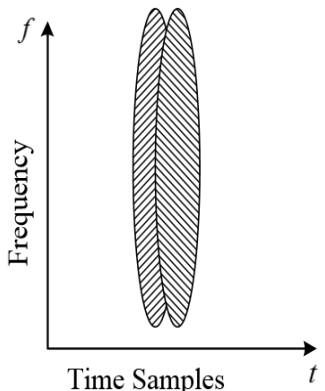

(b)
Fig. 1. TF distribution condition of LFM signals. (a) Tiny non-disjoint LFM signals. (b) Largely non-disjoint LFM signals.

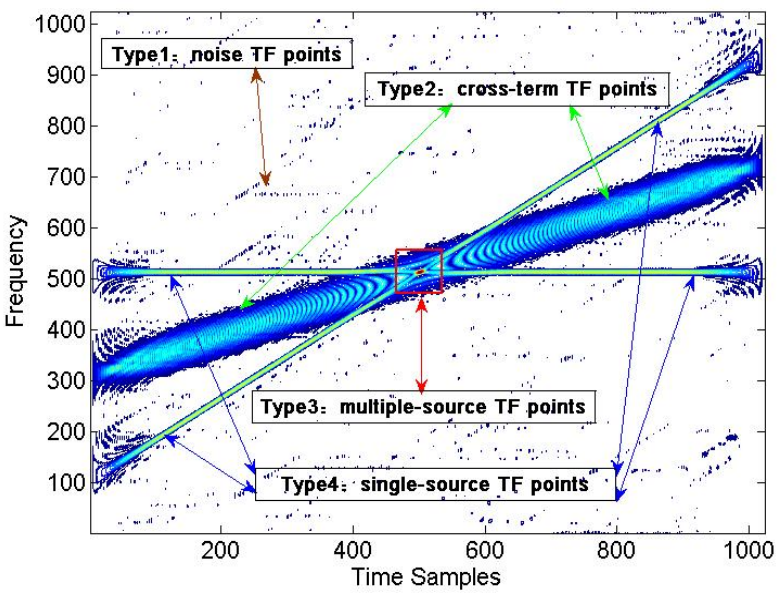

Fig. 2. Different kinds of TF points distribution (Type1: noise TF points, Type2: cross-term TF points, Type3: multiple-source TF points, Type4: single-source $\mathrm{TF}$ points).

TF points, according to [6], only Type4 are required for subsequent DOA estimation and the rest are distracters.

In order to improve the SNR of TF distribution, $\mathbf{W V D}=\sum_{i=1}^{M} \sum_{j=1}^{M} \mathbf{W V D}_{\mathrm{x}_{i} \mathrm{X}_{j}}$ is used for $\mathrm{TF}$ analysis. In the following section, the noise-term, cross-term and multiplesource TF points are eliminated in turn, and the singlesource TF points belonging to each signal can be obtained. Then the STFD matrix is constructed and DOA estimation is achieved.

\subsection{Obtaining Auto-term TF Points}

Since LFM signals have ideal energy aggregation effect, $\mathbf{D}_{\mathrm{XX}}$ is very large at the TF ridgeline and $\mathbf{D}_{\mathrm{XX}}$ is close to zero at other TF points. Let $\Omega_{\mathrm{X}}$ be the TF support domain of $\mathbf{X}(t)$, which satisfies

$$
\begin{array}{ll}
\mathbf{D}_{\mathrm{XX}}(t, f) \neq 0 & (t, f) \in \Omega_{\mathrm{X}}, \\
\mathbf{D}_{\mathrm{XX}}(t, f)=0 & (t, f) \notin \Omega_{\mathrm{X}} .
\end{array}
$$

By filtering out the noise TF points, we can obtain the TF points which have enough energy. One way to do this is as follows: for each sampling time-slice $(t, f)$ of $\mathbf{D}_{\mathrm{XX}}$, the 
following criteria can be performed for all frequency points. We can obtain new TF points set $\Omega_{\mathrm{CA}}$, which includes auto-term TF points and cross-term TF points.

$$
\frac{\left\|\mathbf{D}_{\mathrm{XX}}\left(t_{p}, f_{q}\right)\right\|_{\mathrm{F}}}{\max _{f}\left\|\mathbf{D}_{\mathrm{XX}}\left(t_{p}, f\right)\right\|_{\mathrm{F}}}>\varepsilon_{1} \quad\left(t_{p}, f_{q}\right) \in \Omega_{\mathrm{X}}
$$

where $\|\cdot\|_{F}$ is the Frobenius norm and $\varepsilon_{1}$ is the empirical threshold value, typically, $\varepsilon_{1}=0.05$ when $S N R=10 \mathrm{~dB}$ [22].

In order to eliminate the cross-term TF points, we use the pre-whitening processing method [14]. For each TF point $(t, f), \boldsymbol{\Sigma}$ and $\mathbf{V}$ are eigenvalues and eigenvectors of the STFD matrix respectively. Defining $\mathbf{W}=\boldsymbol{\Sigma}^{-1 / 2} \mathbf{V}^{\mathrm{H}}$ as the whitening matrix, so that

$$
(\mathbf{W A})(\mathbf{W A})^{\mathrm{H}}=\mathbf{U} \mathbf{U}^{\mathrm{H}}=\mathbf{I}
$$

where $(\cdot)^{\mathrm{H}}$ is the conjugate transpose operator. $\mathbf{U}=\mathbf{W A}$ is $N \times N$ unitary matrix and $\mathbf{I}$ stands for the identity matrix. Pre-multiplying and post-multiplying (8) by $\mathbf{W}$, we can get the whitened STFD matrix $\tilde{\mathbf{D}}_{\mathrm{XX}}(t, f)$

$$
\begin{aligned}
\tilde{\mathbf{D}}_{\mathrm{XX}}(t, f) & =\mathbf{W D}_{\mathrm{XX}}(t, f) \mathbf{W}^{\mathrm{H}}=\mathbf{W} \mathbf{A} \mathbf{D}_{\mathrm{SS}}(t, f) \mathbf{A}^{\mathrm{H}} \mathbf{W}^{\mathrm{H}} \\
= & \mathbf{U D}_{\mathrm{SS}}(t, f) \mathbf{U}^{\mathrm{H}} .
\end{aligned}
$$

If $\left(t_{\mathrm{c}}, f_{\mathrm{c}}\right) \in \Omega_{\mathrm{CA}}$ is the cross-term TF point, then there are

$$
\operatorname{trace}\left\{\tilde{\mathbf{D}}_{\mathrm{XX}}\left(t_{\mathrm{c}}, f_{\mathrm{c}}\right)\right\}=\operatorname{trace}\left\{\mathbf{U} \mathbf{D}_{\mathrm{SS}}\left(t_{\mathrm{c}}, f_{\mathrm{c}}\right) \mathbf{U}^{\mathrm{H}}\right\} \approx 0
$$

where trace $\{\cdot\}$ denotes the trace operator. By setting an appropriate threshold value, the cross-term TF points can be eliminated. Thus, we can obtain auto-term TF points set $\Omega_{\mathrm{A}}$

$$
\frac{\operatorname{trace}\left\{\tilde{\mathbf{D}}_{\mathrm{XX}}(t, f)\right\}}{\left\|\tilde{\mathbf{D}}_{\mathrm{XX}}(t, f)\right\|_{\mathrm{F}}}>\varepsilon_{2} \quad(t, f) \in \Omega_{\mathrm{A}}
$$

where $\varepsilon_{2}$ is an empirical value and is related to the noise. Typically, $\varepsilon_{2}=0.85$ when $S N R=10 \mathrm{~dB}$ [23].

\subsection{Extracting Single-source TF Points}

At the signal intersections, signals are coherent owing to same frequency, which brings the rank deficiency of the signal subspace matrix. If the intersections are selected to construct STFD matrix, then subsequent DOA estimation will fail. Therefore, our goal is to remove multiple-source TF points and obtain single-source TF points set belonging to each signal.

In theory, two LFM signals only have one intersection in TF domain. However, since the sampling data is discrete and finite, the TF distribution is somewhat wide, which is called the TF ridgeline. TF ridgeline reflects energy aggregation effect of LFM signals. So there exist multiple TF points around the intersection. In order to obtain singlesource TF points, multiple-source TF points need to be removed according to the empirical threshold value.

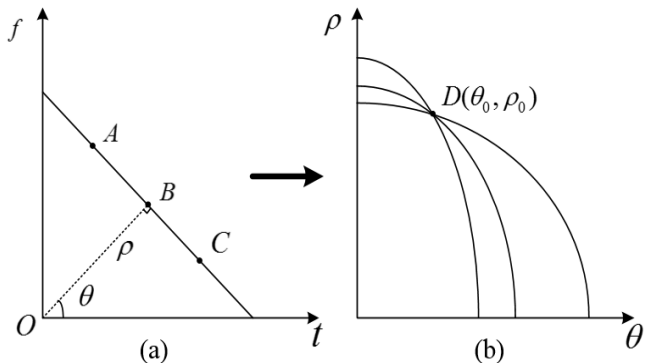

Fig. 3. The Hough transform schematic. (a) Plane coordinate domain. (b) Parameter coordinate domain.

Hough transform detects straight lines by using the voting algorithm in TF domain. The basic idea is to map the plane coordinate system into the parametric coordinate, which makes the mapped results easier to detect.

$$
F\left((t, f),\left(a_{1}, a_{2}, \cdots, a_{m}\right)\right)=0 .
$$

In the plane coordinate system, the line satisfies specific parameters $\left(a_{1}, a_{2}, \ldots, a_{n}\right)$ and $(t, f)$ is located at the line. After mapping into the parameter domain, $\rho$ is the distance between the line and the coordinate origin and $\theta$ is the angle between $\rho$ and sampling time axis. There exists the following relationship

$$
\rho=t \cos (\theta)+f \sin (\theta) .
$$

Equation (18) shows that a point in the plane coordinate system corresponds to a curve in the parameter coordinate system. The different points of the same line in the plane coordinate system intersect at a point in the parameter coordinate system, as is shown in Fig. 3.

Figure 3 shows that three points A, B, C of the same line in the plane coordinate domain can be mapped into three curves and the curves intersect at one point D. By integrating along with different $\theta$, we can obtain the Hough transform. Therefore, Hough transform is a kind of projection integral essentially.

After making the Hough transform, there exist many curves in the parameter coordinate domain and these curves have multiple intersections. We can set appropriate threshold value and carry accumulation statistics, then $P$ peak points can be searched, which are denoted as $\left(\theta_{1}, \rho_{1}\right), \ldots,\left(\theta_{P}, \rho_{P}\right) . P$ peak points correspond to $P$ line segments in the plane coordinate domain.

Assuming that $P$ line segments in TF domain have $Q$ $(Q \leq P(P+1) / 2)$ intersections $\left(t_{1}, f_{1}\right),\left(t_{2,} f_{2}\right), \ldots,\left(t_{Q}, f_{Q}\right)$, then the mathematical equation which each line segment satisfies can be calculated based on the endpoint coordinate

$$
\left\{\begin{array}{c}
f=k_{1} t+b_{1} \\
f=k_{2} t+b_{2} \\
\vdots \\
f=k_{N} t+b_{N}
\end{array}\right.
$$

where $k_{1}, k_{2}, \ldots, k_{N}$ and $b_{1}, b_{2}, \ldots, b_{N}$ are slopes and intercepts of lines, respectively. 
Assuming that there are $n$ signals at the intersection $\left(t_{q}, f_{q}\right)$, multiple-source TF points can be removed by setting threshold value $\varepsilon_{3}$. Thus, we can obtain single-source TF points set $\Omega_{\mathrm{S}}=\cup \Omega_{i}, i=1,2, \ldots, n$ of all LFM signals, and $\Omega_{i}$ denotes the TF points set of the $i$ th signal. The threshold value $\varepsilon_{3}$ is related to the snapshots $L_{\mathrm{S}}$ and sampling rate $f_{\mathrm{S}}$ and initial frequency $f_{0}$ and frequency modulation rate $\gamma$ of LFM signals. When the incident signals and sampling rate are determined, only snapshots can affect $\varepsilon_{3}$.

In order to completely remove multiple-source TF points and reserve as many single-source $\mathrm{TF}$ points as possible, for two LFM signals in Fig. 4, we can quantitatively calculate the empirical threshold value $\varepsilon_{3}$ by using the statistics experiment method. We consider that two LFM signals are non-disjoint in TF domain, which means that the number of signals is two. By traversing all autoterm TF points, the rank of the signal subspace matrix can be solved at each TF point, which is defined as $R$. We know that $R$ is equal to the number of signals at the singlesource TF points and $R$ is less than the number of signals at the multiple-source TF points. Therefore, we count the number of $R=2$ in TF domain, i.e., the number of singlesource TF points, which is denoted as $N_{S}$. Similarly, we can also count the number of $R=1$ in TF domain, i.e., the number of multiple-source TF points, which is denoted as $N_{M}$. $N_{S}$ and $N_{M}$ are standard quantities in the free of noise condition.

Let the threshold value $\varepsilon_{3}$ gradually increases under different SNR, we count the number of single-source TF points $N_{s}$ and the number of multiple-source TF points $N_{m}$. Then successful removing rate of multiple-source $\mathrm{TF}$ points $P_{m}$ and information loss rate of single-source TF points $P_{s}$ are defined along with different SNR and $\varepsilon_{3}$.

$$
P_{m}=\frac{N_{M}-N_{m}}{N_{M}}, P_{s}=\frac{N_{S}-N_{s}}{N_{S}} .
$$

When the SNR is $5 \mathrm{~dB}, 10 \mathrm{~dB}, 15 \mathrm{~dB}$ respectively and $\varepsilon_{3}$ vary from $1 \%$ to $10 \%$, we calculate $P_{m}$ and $P_{s}$ versus different SNR and $\varepsilon_{3}$ by carrying 1000 times Monte Carlo simulations, and the results are shown in Fig. 4. In Fig. 4(a), with the increase of the threshold value $\varepsilon_{3}, P_{m}$ rapidly increases. $P_{m}$ is close to 1 . When $\varepsilon_{3}=6 \%$, which means that multiple-source TF points are completely removed. In addition, the higher SNR is, the larger $P_{m}$ is at the same $\varepsilon_{3}$, which means better removing effectiveness of multiple-source TF points. In Fig. 4(b), with the increase of threshold value $\varepsilon_{3}, P_{s}$ firstly remains unchanged and then slowly increases, which is consistent with the practical situation. Because there are multiple-source TF points at the intersections. Only $\varepsilon_{3}$ increase to somewhat size, singlesource TF points will be loss. What is more, due to $P_{s}$ is not sensitive to the $\mathrm{SNR}$, so $P_{s}$ is basically same at different SNR. In this paper, the threshold value $\varepsilon_{3}$ needs to satisfy the case that $P_{m}=100 \%$ and $P_{s}$ is as small as possible. If single-source TF points are overmuch removed, it has little impact on DOA estimation. Once multiple-source TF points are not completely removed, which will result in the

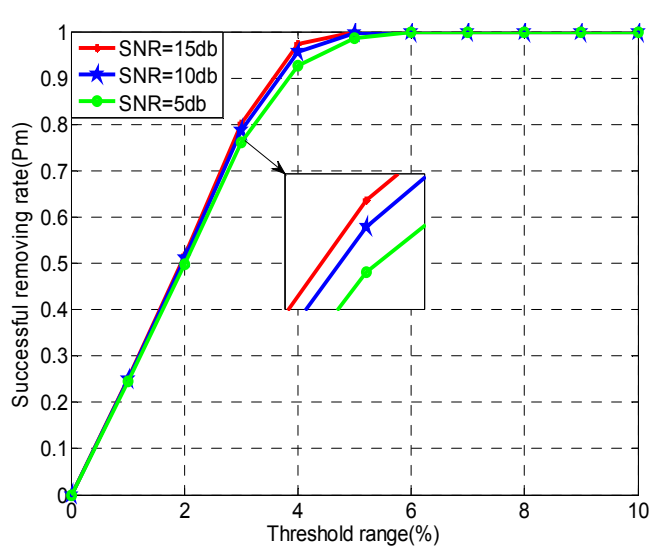

(a) Successful removing rate of multiple-source TF points

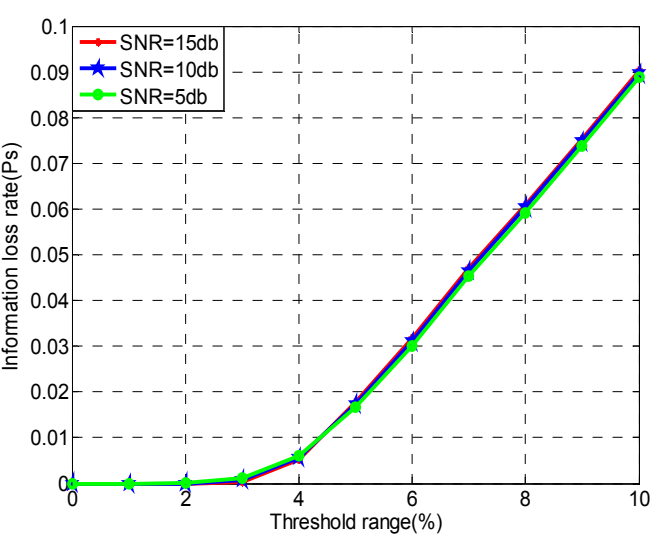

(b) Information loss rate of single-source TF points.

Fig. 4. Calculating the empirical threshold value $\varepsilon_{3}$.

failure of DOA estimation. So we take the empirical threshold value as $\varepsilon_{3}=6 \%$ when $\mathrm{SNR}=10 \mathrm{~dB}$. In the following simulation analysis, we will verify the correctness of $\varepsilon_{3}=6 \%$ in the DOA estimation. Due to the TF energy aggregation effect of WVD, no matter how the initial frequency and modulated frequency of LFM signal change, $\varepsilon_{3}=6 \%$ can be applied to all WVD. Therefore, in the practical application, we remove multiple-source TF points in the set $\left[t_{q}-\varepsilon_{3} L_{s}, t_{q}+\varepsilon_{3} L_{s}\right]$ and obtain single-source TF points set.

\subsection{Constructing STFD Matrix}

For the signal STFD matrix, the diagonal elements have a larger value and the remaining elements are close to zero at the single-source TF points [14]. Therefore, based to (8), the single-source TF points set $\Omega_{i}$ of each signal satisfies

$$
\mathbf{D}_{X X}(t, f)=\mathbf{D}_{S_{i} S_{i}}(t, f) \mathbf{a}_{i} \mathbf{a}_{i}{ }^{H} \quad \forall(t, f) \in \Omega_{i} .
$$

For two TF points $\left(t_{j}, f_{j}\right)$ and $\left(t_{k}, f_{k}\right)$ in $\Omega_{i}$, equation (21) can be written as

$$
\begin{aligned}
& \mathbf{D}_{\mathrm{XX}}\left(t_{j}, f_{j}\right)=\mathbf{D}_{S_{i} S_{i}}\left(t_{j}, f_{j}\right) \mathbf{a}_{i} \mathbf{a}_{i}{ }^{\mathrm{H}}, \\
& \mathbf{D}_{\mathrm{XX}}\left(t_{k}, f_{k}\right)=\mathbf{D}_{S_{i} S_{i}}\left(t_{k}, f_{k}\right) \mathbf{a}_{i} \mathbf{a}_{i}{ }^{\mathrm{H}} .
\end{aligned}
$$

Equation (22) indicates the STFD matrix $\mathbf{D}_{\mathrm{XX}}$ of different TF points of in $\Omega_{i}$ have same eigenvector $\mathbf{a}_{i}$. For any 
TF point $\left(t_{p}, f_{p}\right)$ of $\Omega_{i}$, we can calculate the main eigenvalue $\lambda\left(t_{p}, f_{p}\right)$ and corresponding principal eigenvector $\mathbf{a}\left(t_{p}, f_{p}\right)$ from the STFD matrix $\mathbf{D}_{\mathrm{Xx}}$. Without loss of generality, we can make the first element of $\mathbf{a}\left(t_{p}, f_{p}\right)$ real and positive, namely

$$
\tilde{\mathbf{a}}\left(t_{p}, f_{p}\right)=\left[\begin{array}{c}
\frac{\mathbf{a}_{1}\left(t_{p}, f_{p}\right)}{\left\|\mathbf{a}\left(t_{p}, f_{p}\right)\right\|} \\
\vdots \\
\frac{\mathbf{a}_{M}\left(t_{p}, f_{p}\right)}{\left\|\mathbf{a}\left(t_{p}, f_{p}\right)\right\|}
\end{array}\right] \cdot \frac{\left\|\mathbf{a}_{1}\left(t_{p}, f_{p}\right)\right\|}{\mathbf{a}_{1}\left(t_{p}, f_{p}\right)}
$$

where $\mathbf{a}_{m}\left(t_{p,} f_{p}\right)$ denotes the $m$ th element of $\mathbf{a}\left(t_{p}, f_{p}\right)$.

For single-source TF points set $\Omega_{S}$, mathematically, we decide any two TF points $\left(t_{i}, f_{i}\right)$ and $\left(t_{j}, f_{j}\right)$ to belong to the same signal if they have the following relationship

$$
\mathrm{d}\left(\tilde{\mathbf{a}}\left(t_{i}, f_{i}\right), \tilde{\mathbf{a}}\left(t_{j}, f_{j}\right)\right)<\varepsilon_{4}
$$

where $\mathrm{d}(\cdot)$ is the distance operator, which denotes the Euclidean distance between $\left(t_{i}, f_{i}\right)$ and $\left(t_{j}, f_{j}\right) . \varepsilon_{4}$ is an empirical threshold. Typically, $\varepsilon_{4}=0.05$ when $S N R=10 \mathrm{~dB}$ [14]. By traversing the entire set $\Omega_{S}$, we can obtain the singlesource TF points $\Omega_{i}$ of each signal.

In order to make full use of the single-source TF points, we calculate the averaged STFD matrix $\overline{\mathbf{D}}_{i}(t, f)$

$$
\overline{\mathbf{D}}_{i}(t, f)=\frac{1}{\sum \Omega_{i}} \sum_{(t, f) \in \Omega_{i}} \mathbf{D}_{\mathrm{XX}}(t, f) \quad i=1,2, \cdots, N
$$

where $\Sigma \Omega_{i}$ are the number of single-source TF points in $\Omega_{i}$. $\overline{\mathbf{D}}_{i}(t, f)$ is similar to the covariance matrix in the subspacebased algorithm. So we construct spectrum search function and carry DOA estimation based on the MUSIC algorithm.

$$
\mathrm{P}(\theta)=\frac{1}{\mathbf{a}^{\mathrm{H}}(\theta) \mathbf{U}_{\mathrm{n}} \mathbf{U}_{\mathrm{n}}{ }^{\mathrm{H}} \mathbf{a}(\theta)}
$$

where $\mathbf{a}(\theta, \varphi)$ is steering vector and $\mathbf{U}_{\mathrm{n}}$ is the noise subspace.

For the readers' convenience, the procedure of singlesource TF points selection algorithm based on Hough transform is summarized as follows:

(1) Filter out noise based on empirical threshold value $\varepsilon_{1}$.

(2) Eliminate cross-term TF points of WVD based on pre-whitening algorithm and obtain auto-term TF points.

(3) Remove multiple-source TF points based on Hough transform and obtain single-source TF points.

(4) Classify signals according to the Euclidean distance operator and get single-source TF points $\Omega_{i}$ of each signal.

(5) For every signal, calculate the averaged STFD matrix $\overline{\mathbf{D}}_{i}(t, f)$ in $\Omega_{i}$, then construct the spectrum search function $\mathrm{P}(\theta)=1 /\left(\mathbf{a}^{\mathrm{H}}(\theta) \mathbf{U}_{\mathrm{n}} \mathbf{U}_{\mathrm{n}}{ }^{\mathrm{H}} \mathbf{a}(\theta)\right)$ based on the MUSIC algorithm.

\section{Simulation and Results}

In order to verify the performance of the proposed algorithm, the following simulations are carried. We consider that three spectrally-overlapped LFM signals come from angles: $-20^{\circ}, 0^{\circ}$ and $20^{\circ}$. The ULA has 8 elements and array element spacing $d$ is half of wavelength, which corresponds to the lowest frequency of LFM signals. The sampling rate is $360 \mathrm{MHz}$ and snapshots are 512 . The frequency distribution of three signals are $[80,140] \mathrm{MHz}$, $[20,100] \mathrm{MHz},[160,30] \mathrm{MHz}$ respectively, and all signals have normalized amplitudes. The proposed algorithm is implemented by a PC with AMD Phenom ${ }^{\text {TM }}$ IIX6 1055T Processor $2.8 \mathrm{GHz} \mathrm{CPU}$ and $8 \mathrm{~GB}$ RAM by running the MATLAB codes. Due to the fact that the calculations of Hough transform have been integrated as the functions of MATLAB, we just need to use these functions when we implement the proposed algorithm.

\subsection{The TF Distribution of Multiple LFM Signals}

Assuming there are two intersections between three LFM signals, then the TF distribution is shown in Fig. 5(a). Firstly, we apply the proposed algorithm to remove crossterm TF points, and we can obtain auto-term TF points distribution in Fig. 5(b). According to the analysis of Fig. 4, multiple-source TF points can be eliminated based on $\varepsilon_{3}=6 \%$. Then we can obtain single-source TF points distribution in Fig. 5(c). Finally, single-source TF points of each signal can be classified by using the Euclidean distance operator, as is shown in Fig. 5(d).

\subsection{The Analysis of Empirical Threshold Value}

The proposed algorithm involves four empirical threshold values. The threshold value $\varepsilon_{1}$ eliminates the effect of noise in TF domain, which is related to the SNR. We take $\varepsilon_{1}=0.05$ when $\mathrm{SNR}=10 \mathrm{~dB}$, and $\varepsilon_{1}$ has been verified by [22]. The threshold value $\varepsilon_{2}$ removes cross-term TF points. The bigger $\varepsilon_{2}$ is, the more complete cross-term $\mathrm{TF}$ points will be eliminated. But too big $\varepsilon_{2}$ will result in the loss of the auto-term TF points. Therefore, we take $\varepsilon_{2}=0.85$ when $\mathrm{SNR}=10 \mathrm{~dB}$ in this paper and $\varepsilon_{2}$ has been verified by [23]. Typically, $\varepsilon_{4}=0.05$, which is also verified by [14]. This paper firstly proposes the concept of threshold value $\varepsilon_{3}$, in order to completely remove multiplesource TF points and reserve as many single-source TF points as possible, this paper uses the statistical experiment method. In the above analysis, we take $\varepsilon_{3}=6 \%$. Multiplesource TF points may be residual when $\varepsilon_{3}$ is too small, which will result in the failure of DOA estimation. When $\varepsilon_{3}$ is too big, the algorithm can correctly estimate the DOA. However, the loss of single-source TF points will bring the reduction of SNR. Figure 6(a), 6(b) and 6(c) simulate the spatial distribution of DOAs when $\varepsilon_{3}=4 \%, \varepsilon_{3}=6 \%$ and $\varepsilon_{3}=10 \%$, respectively. It can be seen from Fig. 6 that the 


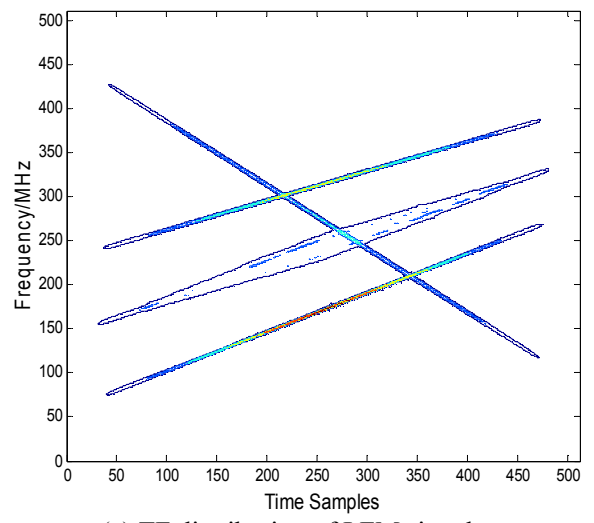

(a) TF distribution of LFM signals.

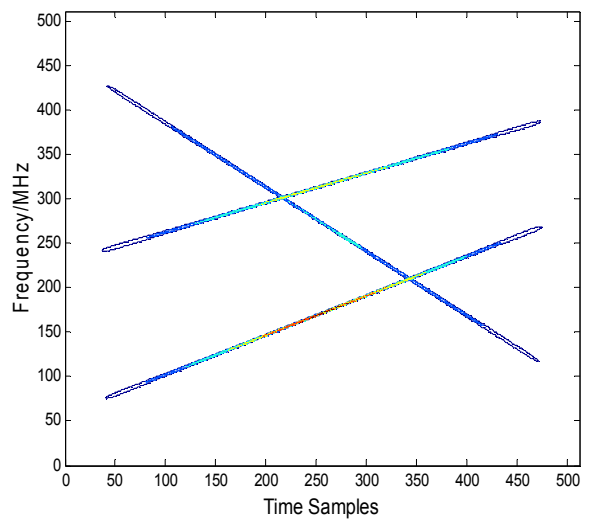

(b) Auto-term TF points distribution.

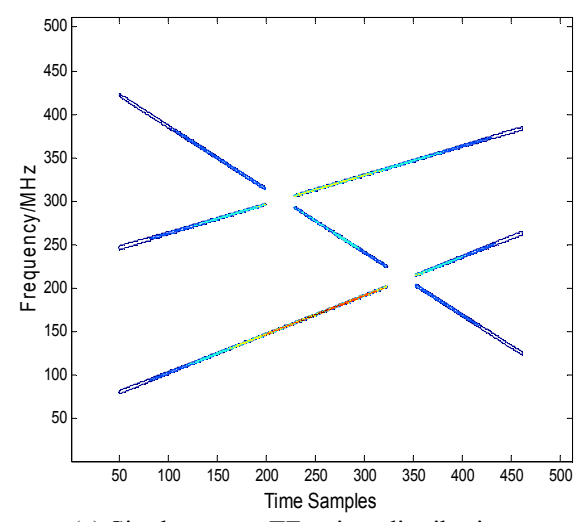

(c) Single-source TF points distribution.

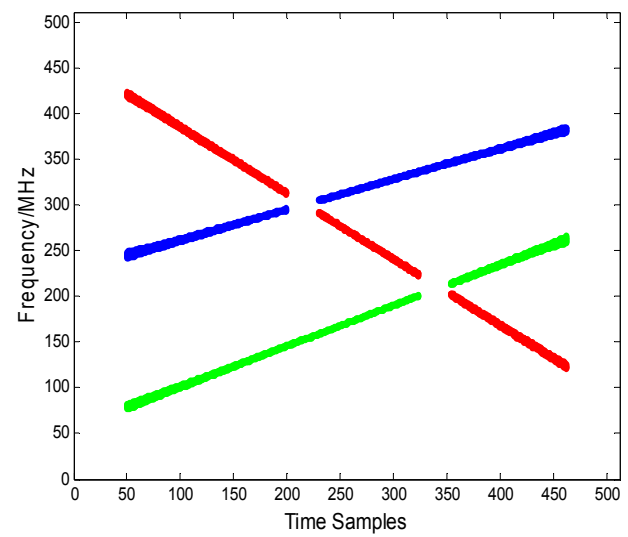

(d) Single-source TF points distribution of each signal.

Fig. 5. TF distribution of three LFM signals.
DOA estimation will be wrong when $\varepsilon_{3}=4 \%$, since multiple-source TF points are not completely removed, which is consistent with the analysis in Fig. 4(a). When $\varepsilon_{3}=6 \%$ or $\varepsilon_{3}=10 \%$, correct DOA estimation all can be achieved, but Figure 6(b) has stronger spectral peak energy aggregation. Therefore, in order to obtain better SNR, we generally take $\varepsilon_{3}=6 \%$ in the practical application.

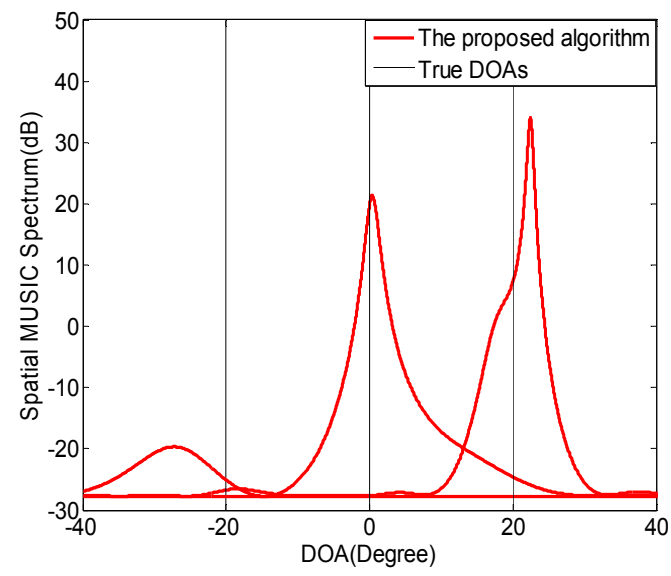

(a) $\varepsilon_{3}=4 \%$

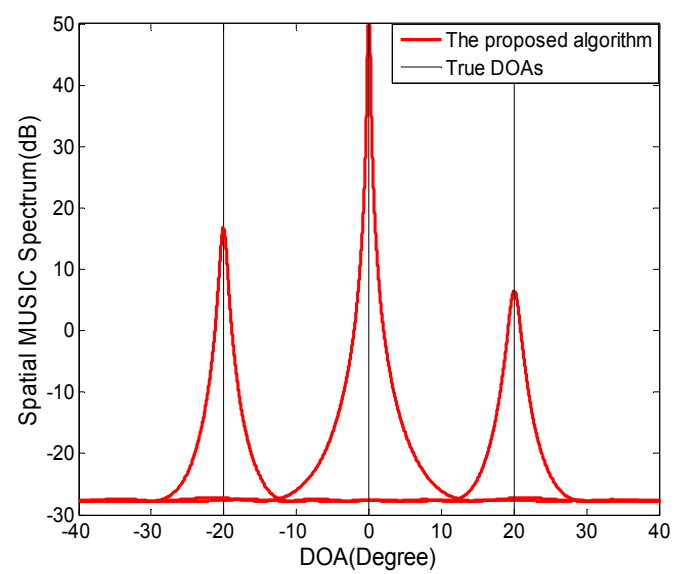

(b) $\varepsilon_{3}=6 \%$

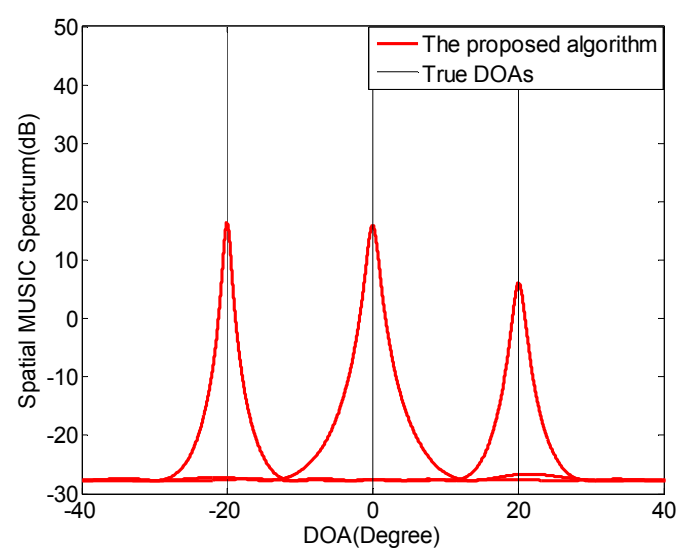

(c) $\varepsilon_{3}=10 \%$

Fig. 6. Spatial spectra distribution versus threshold value $\varepsilon_{3}$. 


\subsection{The Spatial Angular Resolution and DOA Estimation Accuracy Analysis of Different Algorithms}

In order to analyze the angular resolution and DOA estimation accuracy of the proposed algorithm, the comparison between the proposed algorithm, the TF-MUSIC algorithm [8] and the TF-FBSS-MUSIC algorithm [19] are presented in Fig. 7 and Fig. 8. The cross-term TF points of TF-MUSIC algorithm and TF-FBSS-MUSIC algorithm are also eliminated based on the pre-whitening algorithm [14]. In addition, we assume the TF points of the TF-MUSIC algorithm are available. We select the TF points at the TF ridgeline to estimate DOAs. We consider that two spectrally-overlapped LFM signals come from angles: $-5^{\circ}$ and $5^{\circ}$. The frequency distribution of two signals are $[80,140] \mathrm{MHz},[160,30] \mathrm{MHz}$ respectively. The spatial spectrum distribution of three algorithms is shown in Fig. 7(a). It can be seen that three algorithms all can achieve correct DOA estimation, and the proposed algorithm has the strongest spectral peak energy aggregation. In order to further analyze DOA estimation precision, the root-mean-square error (RMSE) is introduced. $M$ stands for the number of Monte Carlo simulations and $N$ is the number of signals. $\hat{\theta}_{m n}$ is the estimated value of $\theta_{n}$ in the $m$ th simulation.

$$
R M S E_{\mathrm{DOA}}=\sqrt{\sum_{m=1}^{M} \sum_{n=1}^{N}\left(\hat{\theta}_{m n}-\theta_{n}\right)^{2} / M N}
$$

The Cramer-Rao Lower Bound (CRB) is also introduced in following simulations [25]. We carry 1000 times Monte Carlo simulations and Figure 7(b) shows that the DOA estimation RMSE of two LFM signals along with SNR when the array element spacing is $10^{\circ}$. We can see from Fig. 7(b) that the RMSE of three algorithms decrease rapidly with the increase of the SNR. In general, the TFFBSS-MUSIC algorithm has the worst estimation precision, followed by the TF-MUSIC algorithm. The proposed algorithm has the highest DOA estimation precision. Because the TF-FBSS-MUSIC is a kind of dimension reduction algorithm due to perform the spatial smoothing algorithm, which results in the loss of the array aperture. As a result, the estimation accuracy is reduced. The TF-MUSIC algorithm performs DOA estimation only by using the TF points at the TF ridgeline. When the SNR is larger, the estimation precision is close to the proposed algorithm. However, when the SNR is smaller, the estimation accuracy is poor owing to the distracters. In addition, it is not realistic to assume that the TF points are available at the TF ridgeline. The proposed algorithm extracts single-source TF points of each signal by removing the cross-term TF points and multiple-source TF points, and calculates the averaged STFD matrix of the all TF points belonging to each signal. So the proposed algorithm has the best DOA estimation precision.

The incident angles are reduced to $-0.5^{\circ}$ and $0.5^{\circ}$, and other simulation conditions remain unchanged. The spatial spectrum distribution of the proposed algorithm is shown in Fig. 8(a) when SNR is $10 \mathrm{~dB}$. It can be seen that the proposed algorithm can still distinguish two signals. The mainly reason is that the single-source TF points of each signal are extracted based on Hough transform. Although the signals are closely-spaced, they are still distinctly distributed in the TF domain. In the same way, the RMSE versus SNR is shown in Fig. 8(b). We can see that the proposed algorithm still has the best DOA estimation precision. When the signal spacing is smaller or the SNR is lower, the estimation performance will become poor.

According to the simulation results of Fig. 7 and Fig. 8, we can see that the proposed algorithm has good angular resolution, which can up to $1^{\circ}$ when SNR is $10 \mathrm{~dB}$. Compared with the TF-MUSIC algorithm and the TFFBSS-MUSIC algorithm, the proposed algorithm has higher DOA estimation accuracy. We consider that the ULA has $M$ sensors and $N$ far-field LFM signals imping on the ULA. The snapshots are $L_{\mathrm{S}}$. In the TF-FBSS-MUSIC algorithm, we assume that the sub-array elements are $P$ $(P<M)$. The computational complexity of several algorithms is analyzed in Tab. 1. We can see from Tab. 1 that the TF-FBSS-MUSIC algorithm has the lowest computation

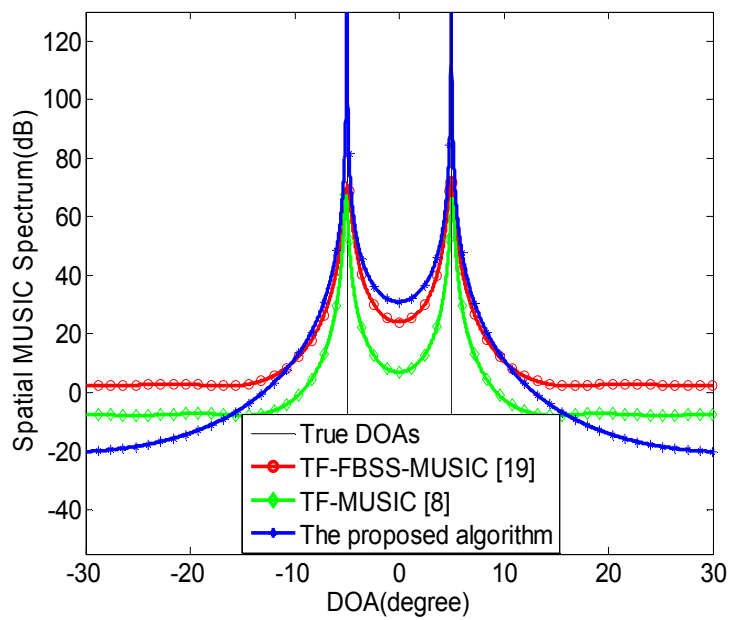

(a) Spatial spectra distribution of three algorithms.

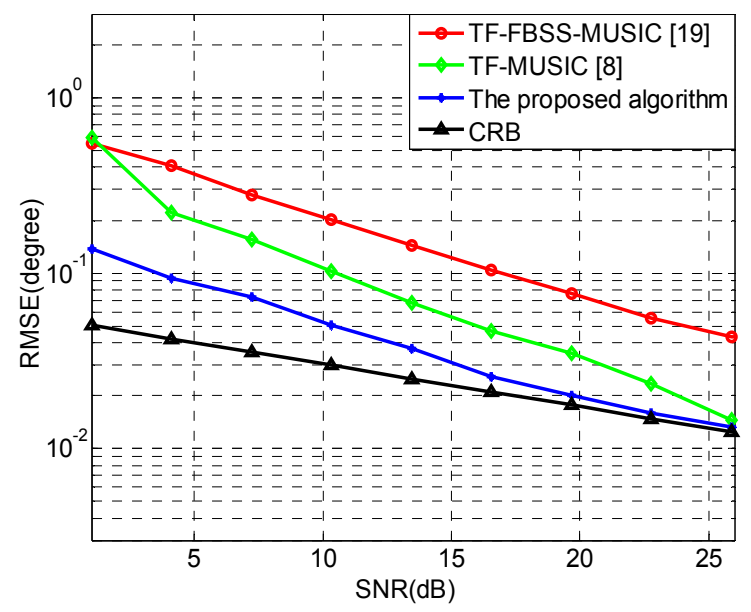

(b) The RMSE of DOAs estimation versus SNR.

Fig. 7. The estimation results (DOA spacing: $10^{\circ}$ ). 


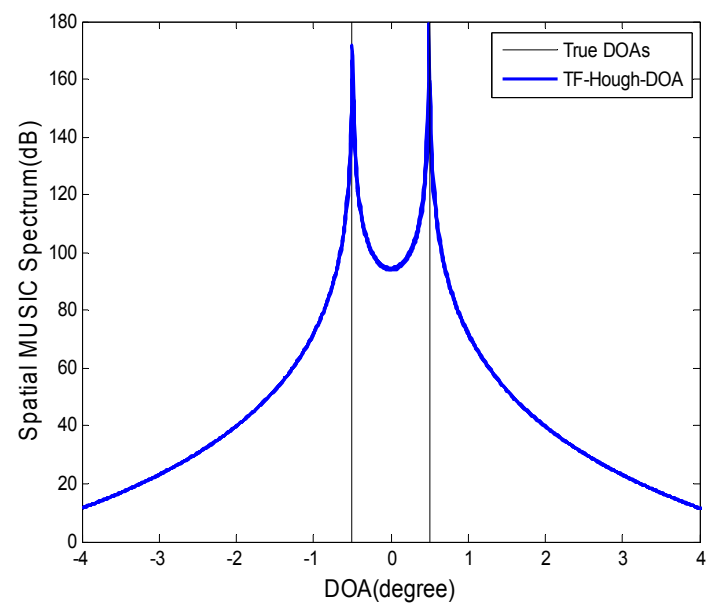

(a) Spatial spectra distribution of proposed algorithm.

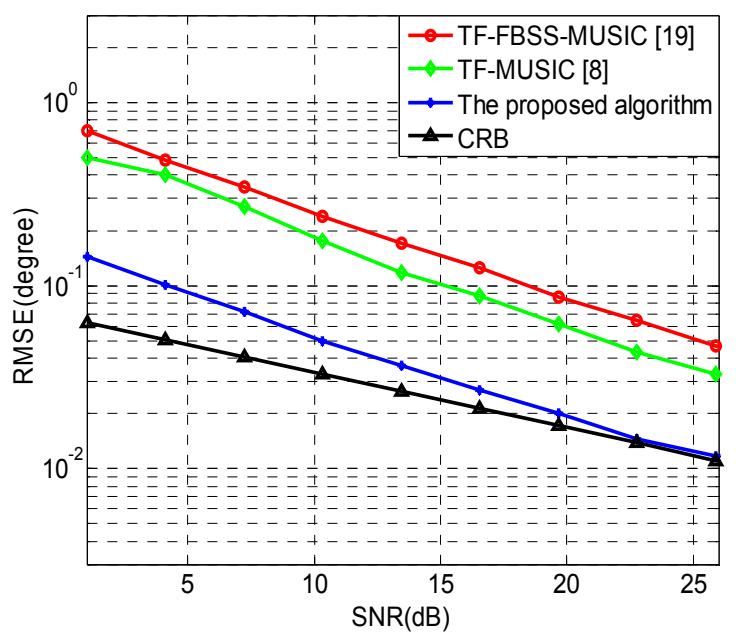

(b) The RMSE of DOAs estimation versus SNR.

Fig. 8. The estimation results (DOA spacing: $1^{\circ}$ ).

\begin{tabular}{|c|c|}
\hline Algorithm & Computation Complexity \\
\hline TF-FBSS-MUSIC [19] & $O\left(N P^{2} L_{\mathrm{S}}^{2} \log _{2} L_{\mathrm{S}}\right)$ \\
\hline TF-MUSIC [8] & $O\left(N M^{2} L_{\mathrm{S}}{ }^{2} \log _{2} L_{\mathrm{S}}\right)$ \\
\hline The proposed method & $O\left(N M^{2} L_{\mathrm{S}} \log _{2} L_{\mathrm{S}}+\Delta\right)$ \\
\hline
\end{tabular}

Tab. 1. Comparison of computation complexity.

complexity. The TF-MUSIC algorithm and the proposed algorithm have similar computational complexity. $\Delta$ is the increased calculation owing to select the single-source TF points and $\Delta$ is much smaller than $N M^{2} L_{\mathrm{S}}{ }^{2} \log _{2} L_{\mathrm{S}}$. In summary, the TF-MUSIC algorithm is not practical to assume that the TF points are available at the ridgeline, and the TFFBSS-MUSIC algorithm possesses poor estimation accuracy. In contrast, the proposed algorithm has higher estimation accuracy and acceptable computational complexity, which will have broad application prospects in the engineering.

\section{Experimental Verification}

In order to verify the effectiveness of the proposed method, we carry out the experiment in the standard micro-

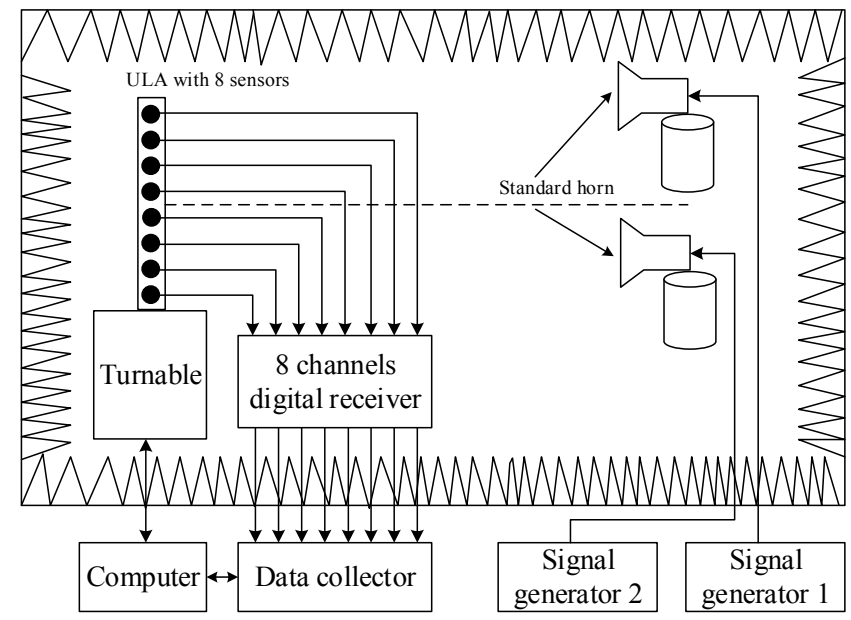

Fig. 9. The sketch map of the experimental platform.

wave anechoic chamber. It should be noted that the reference [19] has constructed the hardware experimental platform, and we use the same platform to verify the proposed algorithm. The sketch map of the platform is shown in Fig. 9.

We consider that the ULA has eight sensors and two LFM signals imping on the ULA. The eight channels digital receiver receives signals from the ULA and stores signals into the data collector, then, the computer can use the proposed algorithm to estimate DOAs of the signals. In the standard microwave anechoic chamber, the turntable is controlled by the computer and the distance between the horn and the turntable is $17 \mathrm{~m}$. The standard horns are respectively located at $-10^{\circ}$ and $10^{\circ}$ relative to the ULA. We use two signal generators to produce two LFM signals whose frequency distributions are $[30,120] \mathrm{MHz}$ and

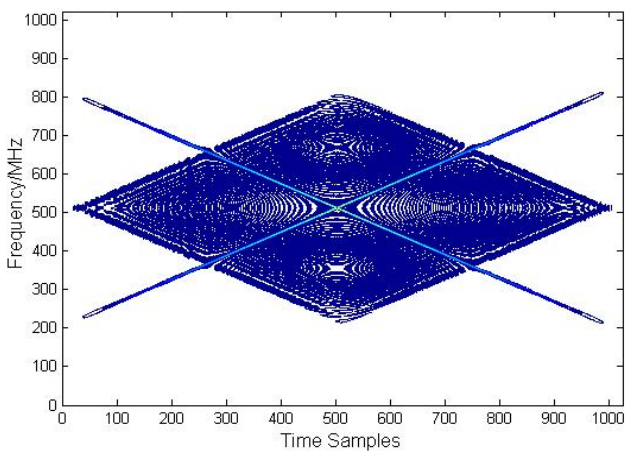

(a) TF distribution after eliminating noise.

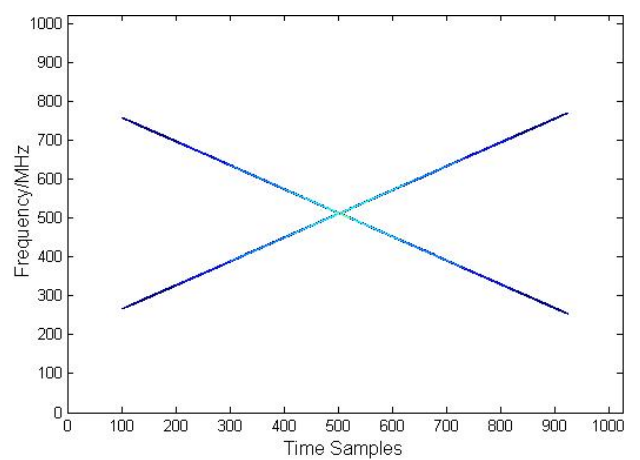

(b) TF distribution after removing cross-term. 


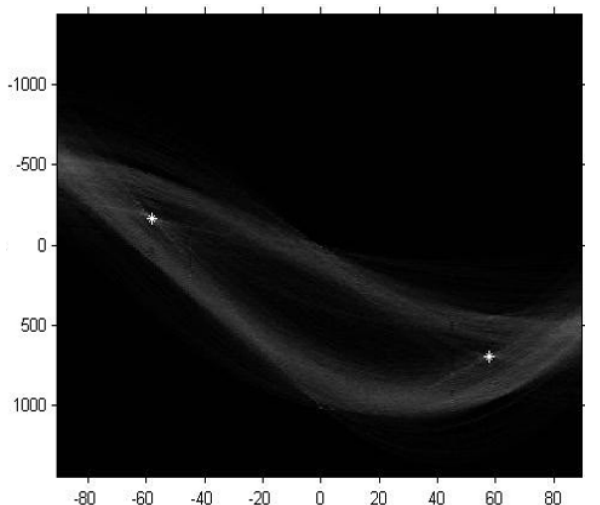

(c) Peak distribution in the parameter domain.

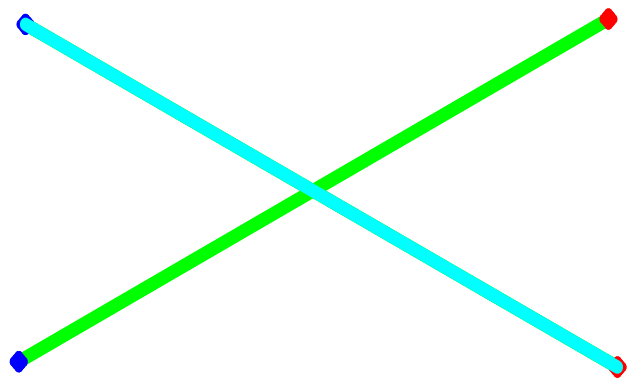

(d) Line detection result based on Hough transform.

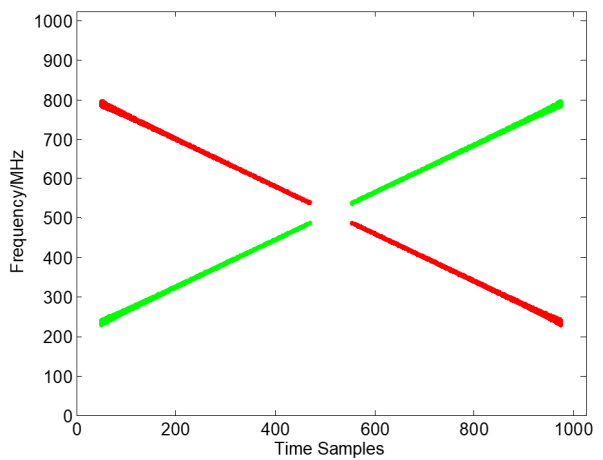

(e) Single-source TF points set of each signal.

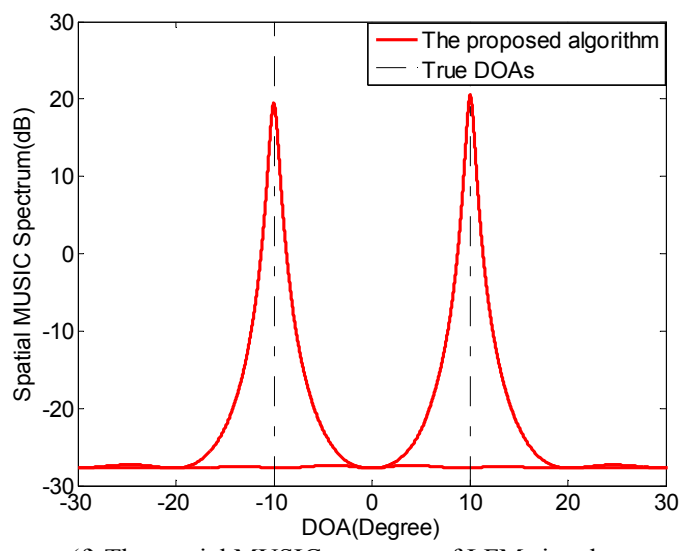

(f) The spatial MUSIC spectrum of LFM signals.

Fig. 10. The sketch map of the experimental platform.

$[120,30] \mathrm{MHz}$, respectively. The amplitude of the signals is $0 \mathrm{dBm}$. The sampling frequency of the data collector is $300 \mathrm{MHz}$ and the sampling snapshots are 1024. Based on the proposed algorithm, the TF distribution results of two LFM signals are shown in Fig. 10.
After eliminating noise, the TF distribution is shown in Fig. 10(a). Based on the pre-whitening algorithm, it can be seen that the cross-term points are basically removed in TF domain, as it is shown in Fig. 10(b). In addition, the TF energy aggregation of LFM signals is pretty good. Figure 10 (c) shows two searched peaks which is indicated by white and Figure 10(d) shows the detected two line segments. The blue and red are endpoints of detected line segments respectively. By removing multiple-source TF points, Figure 10(e) shows the single-source TF points set belonging to each signals. Then, the averaged spatial TF distribution matrix is constructed and DOA estimation is realized based on the multiple signal classification (MUSIC) algorithm. It can be seen from Fig. 10(f) that DOA estimations are $-9.92^{\circ}$ and $10.13^{\circ}$, which are in accordance with the sets. The experimental results show that the proposed algorithm can efficiently deal with the spectrally-overlapped LFM signals and has a broad application prospect.

\section{Conclusion}

In order to achieve the DOA estimation when multiple LFM signals are non-disjoint in TF domain, this paper proposes the single-source TF points selection algorithm based on the WVD and Hough transform. Firstly, we use the threshold value $\varepsilon_{1}$ to filter out the noise. Then we eliminate cross-term TF points of WVD based on the prewhitening algorithm. In order to completely remove multiple-source TF points and reserve as many single-source TF points as possible, this paper firstly proposes the concept of threshold value $\varepsilon_{3}$ by using the statistical experiment method. Since the proposed algorithm can automatically select single-source TF points set belonging to each signal, it has higher spatial resolution and pretty good DOA estimation accuracy compared with existing methods. Although the current algorithm is only applicable to LFM signals which satisfy the straight line distribution in TF domain, generalized Hough transform can detect other forms of signals with different shapes, such as sinusoidal FM signal and frequency-shift-keying signal. Therefore, the proposed algorithm has broad application prospect in the electronic countermeasures.

\section{References}

[1] SCHMIDT, R. O. Multiple emitter location and signal parameter estimation. IEEE Transactions on Antennas and Propagation, 1986, vol. 34, no. 3, p. 276-280. DOI: 10.1109/tap.1986.1143830

[2] ROY, R., PAULRAJ, A., KAILATH, T. Direction-of-arrival estimation by subspace rotation methods-ESPRIT. In Proceedings of the IEEE International Conference on Acoustics, Speech, and Signal Processing (ICASSP). Tokyo (Japan), 1986, p. 2495-2498. DOI: 10.1109 /icassp.1986.1168673

[3] CUI, K., WU, W., CHEN, X., et al. 2-D DOA estimation of LFM signals based on dechirping algorithm and uniform circle array. 
Radioengineering, 2017, vol. 26, no. 1, p. 299-308. DOI: $10.13164 /$ re.2017.0299

[4] CHEN, H., WAN, Q., FAN, R., et al. Direction-of-arrival estimation based on sparse recovery with second-order statistics. Radioengineering, 2015, vol. 24, no. 1, p. 208-213. DOI: $10.13164 /$ re. 2015.0208

[5] GUO, M., MAO, X., LI, S., et al. A fast DOA estimation algorithm based on polarization MUSIC. Radioengineering, 2015, vol. 24, no. 1 , p. 214-225. DOI: $10.13164 /$ re.2015.0214

[6] BELOUCHRANI, A., AMIN, M. G., THIRION-MOREAU, N., et al. Source separation and localization using time-frequency distributions: an overview. IEEE Signal Processing Magazine, 2013, vol. 30, no. 6, p. 97-107. DOI: 10.1109/msp.2013.2265315

[7] BELOUCHRANI, A., AMIN, M. G. Blind source separation based on time-frequency signal representations. IEEE Transactions on Signal Processing, 1998, vol. 46, no. 11, p. 2888-2897. DOI: $10.1109 / 78.726803$

[8] BELOUCHRANI, A., AMIN, M. G. Time-frequency MUSIC. IEEE Signal Processing Letters, 1999, vol. 6, no. 5, p. 109-110. DOI: $10.1109 / 97.755429$

[9] AMIN, M. G., ZHANG, Y. Direction finding based on spatial time-frequency distribution matrices. Digital Signal Processing 2000 , vol. 10 , no. 4 , p. $325-339$. DOI: $10.1006 /$ dspr.2000.0374

[10] ZHANG, Y., MU, W., AMIN, M. G. Subspace analysis of spatial time-frequency distribution matrices. IEEE Transactions on Signal Processing, 2001, vol. 49, no. 4, p. 747-759. DOI: $10.1109 / 78.912919$

[11] YILMAZ, O., RICKARD, S. Blind separation of speech mixtures via time-frequency masking. IEEE Transactions on Signal Processing, 2004, vol. 52, no. 7, p. 1830-1847. DOI: 10.1109/tsp.2004.828896

[12] GERShMAN, A. B., PESAVENTO, M., AMIN, M. G. Estimating parameters of multiple wideband polynomial-phase sources in sensor arrays. IEEE Transactions on Signal Processing, 2001, vol. 49, no. 12, p. 2924-2934. DOI: 10.1109/78.969501

[13] GERSHMAN, A. B., AMIN, M. G. Coherent wideband DOA estimation of multiple FM signals using spatial time-frequency distributions. In Proceedings of the IEEE International Conference on Acoustics, Speech, and Signal Processing (ICASSP). Istanbul (Turkey), 2000, p. 3065-3068. DOI: 10.1109/ icassp.2000.861184

[14] LINH-TRUNG, N., BELOUCHRANI, A., ABED-MERAIM, K. et al. Separating more sources than sensors using time-frequency distributions. EURASIP Journal on Applied Signal Processing, 2005, vol. 17, p. 2828-2847. DOI: 10.1155/ASP.2005.2828

[15] HEIDENREICH, P., CIRILLO, L. A., ZOUBIR, A. M Morphological image processing for FM source detection and localization. Signal Processing, 2009, vol. 89, no. 6 , p. 1070-1080. DOI: 10.1016/j.sigpro.2008.12.011

[16] JOURJINE, A., RICKARD, S., YILMAZ, O. Blind separation of disjoint orthogonal signals: Demixing $\mathrm{N}$ sources from 2 mixtures. In Proceedings of the IEEE International Conference on Acoustics, Speech, and Signal Processing (ICASSP). Istanbul (Turkey), 2000, p. 2985-2988. DOI: 10.1109/icassp.2000.861162

[17] KIM, S. G., YOO, C. D. Underdetermined blind source separation based on subspace representation. IEEE Transactions on Signal Processing, 2009, vol. 57, no. 7, p. 2604-2614. DOI: $10.1109 /$ tsp. 2009.2017570

[18] ZHANG, H., BI, G., CAI, Y., et al. DOA estimation of closelyspaced and spectrally-overlapped sources using a STFT-based MUSIC algorithm. Digital Signal Processing, 2016, vol. 52, no. C, p. 25-34. DOI: $10.1016 /$ j.dsp.2016.01.015

[19] CUI, K., CHEN, X., HUANG, J., et al. DOA estimation of multiple LFM sources using a STFT-based and FBSS-based
MUSIC algorithm. Radioengineering, 2017, vol. 26, no. 4, p. 1126-1137. DOI: $10.13164 /$ re.2017.1126

[20] ZHANG, Y., AMIN, M. G. Blind separations of nonstationary sources based on spatial time-frequency distributions. EURASIP Journal on Advances in Signal Processing, 2006, p. 1-13. DOI: $10.1155 / \mathrm{asp} / 2006 / 64785$

[21] FADAILI, E. M., MOREAU, N. T., MOREAU, E. Nonorthogonal joint diagonalization/zero diagonalization for source separation based on time-frequency distributions. IEEE Transactions on Signal Processing, 2007, vol. 55, no. 5, p. 1673-1687. DOI: 10.1109/tsp.2006.889469

[22] AISSA EL-BEY, A., LINH-TRUNG, N., ABED-MERAIM, K., et al. Underdetermined blind separation of nondisjoint sources in the time-frequency domain. IEEE Transactions on Signal Processing, 2007, vol. 55, no. 3, p. 897-907. DOI: 10.1109/tsp.2006.888877

[23] Belouchrani, A., ABED-MERAIM, K., AMIN, M. G., et al. Joint anti-diagonalization for blind source separation. In Proceedings of the IEEE International Conference on Acoustics, Speech, and Signal Processing (ICASSP). Salt Lake City (USA), 2001, vol. 5, p. 2789-2792. DOI: 10.1109/icassp.2001.940225

[24] WIGNER, E. P. On the quantum correction for thermodynamic equilibrium. Physical. Review, 1932, vol. 40, no. 5, p. 749-759. DOI: $10.1103 /$ physrev.40.749

[25] BABAROSSA, S. Analysis of multicomponent LFM signals by a combined Wigner-Hough transform. IEEE Transaction on Signal Processing, 1995, vol. 43, no. 6, p. 1511-1515. DOI: $10.1109 / 78.388866$

[26] CHAMBERS, C., TOZER, T. C., SHARMAN, K. C., et al. Temporal and spatial sampling influence on the estimates of superimposed narrowband signals: when less can mean more. IEEE Transactions on Signal Processing, 1996, vol. 44, no. 12, p. 3085-3098. DOI: $10.1109 / 78.553482$

\section{About the Authors ...}

Weike ZHANG (corresponding author) was born in 1992. He received his M.S. degree in the College of Electronic Science from the National University of Defense Technology (NUDT) in 2016. His research interests include array signal processing and high-resolution angle measurement algorithms.

Kaibo CUI was born in 1990. He received his Ph.D. degree in the College of Electronic Science, NUDT in 2018. His research interests include signal processing and spatial spectrum estimation.

Weiwei WU was born in 1981. She received her M.S. and $\mathrm{Ph} . \mathrm{D}$. degree in the College of Electronic Science, NUDT, in 2008 and 2011, respectively. Her research interests include array signal processing and antenna design.

Tao XIE was born in 1990. He received his M.S. degree in the College of Electronic Science, NUDT, in 2015. His research interests include radar signal processing and SAR image processing.

Naichang YUAN was born in 1965. He received his M.S. and Ph.D. degree in Electronic Science and Technology from the University Science and Technology of China in 1991 and 1994, respectively. His research interests include array signal processing, signal processing in radar. 